\title{
High-precision scanning transmission electron microscopy at coarse pixel sampling for reduced electron dose
}

\author{
Andrew B Yankovich', Benjamin Berkels², Wolfgang Dahmen ${ }^{3,4}$, Peter Binev ${ }^{3}$ and Paul M Voyles ${ }^{1 *}$
}

\begin{abstract}
Determining the precise atomic structure of materials' surfaces, defects, and interfaces is important to help provide the connection between structure and important materials' properties. Modern scanning transmission electron microscopy (STEM) techniques now allow for atomic resolution STEM images to have down to sub-picometer precision in locating positions of atoms, but these high-precision techniques generally require large electron doses, making them less useful for beam-sensitive materials. Here, we show that 1- to 2-pm image precision is possible by non-rigidly registering and averaging a high-angle dark field image series of a 5- to 6-nm Au nanoparticle even though a very coarsely sampled image and decreased exposure time was used to minimize the electron dose. These imaging conditions minimize the damage to the nanoparticle and capture the whole nanoparticle in the same image. The high-precision STEM image reveals bond length contraction around the entire nanoparticle surface, and no bond length variation along a twin boundary that separates the nanoparticle into two grains. Surface atoms at the edges and corners exhibit larger bond length contraction than atoms near the center of surface facets.
\end{abstract}

Keywords: High precision; STEM; Gold nanoparticle; Image registration; Coarse pixel

\section{Background}

Many materials science problems require relating materials' properties to the atomic structure near the materials' surfaces, interfaces, extended defects, point defects, and other types of strain fields. Transmission electron microscopy (TEM) and scanning TEM (STEM) allow for the imaging of these atomic structures, helping us understand property-structure relationships. With the advent of aberration correctors for electron microscopes, sub-Angstrom atomic resolution is regularly achievable [1]. Once an atomic resolution image of a desired structure is acquired, the question becomes how well are the positions of the atomic columns known? Image spatial precision is a measure of the statistical spread of repeated distance measurements in an image and helps quantify the answer to this question. The best reported precision for TEM images is 1 to $3 \mathrm{pm}[2-4]$ and for STEM images, until recently, was 4

\footnotetext{
* Correspondence: voyles@engr.wisc.edu

'Department of Materials Science and Engineering, University of Wisconsin-Madison, 1509 University Ave, Madison, WI 53706, USA Full list of author information is available at the end of the article
}

to $5 \mathrm{pm}[5,6]$, showing that precision much smaller than the resolution is achievable.

The signal-to-noise ratio (SNR) [7] of an image provides a fundamental limit for the precision, but in practice for STEM, instabilities usually limit the precision well above the SNR limit. The serial acquisition of pixels in STEM puts it at a disadvantage compared to the parallel acquisition of pixels in TEM because serial acquisition translates position instabilities of the probe and sample into displacements of the imaged atoms. Despite these disadvantages, high-angle annular dark field (HAADF) STEM imaging has the advantage of giving a faithful representation of the sample structure over a wide range of thickness and defocus, making it more interpretable and quantifiable than TEM imaging. Techniques have been developed to reduce the effect of the instabilities and increase the image SNR causing an improvement in image precision by averaging many short exposure frames that have been rigidly registered to one another $[3,5]$. However, these techniques only can account for sample drift between frames, leaving the other instabilities still present in STEM images and limiting the image precision. We have 
developed a non-rigid (NR) registration scheme that enables the correction of instabilities in STEM image series at all time and length scales $[8,9]$. NR registration allows for extremely high SNR images by averaging many frames that have strongly reduced distortions, enabling sub-picometer precision measurements of atom positions in aberration-corrected Z-contrast STEM images of singlecrystal materials and measurements of picometer-scale bond length variation at the surface of a Pt nanocatalyst [8]. It also enables standardless atom counting [10] with the best reported uncertainty by uniquely determining the number of atoms in a column in $60 \%$ of the imaged columns [8].

The primary drawback to our high-precision STEM technique is the large dose resulting from the large amount of images required to get the increased SNR. This makes high-precision imaging either difficult or impossible for beam-sensitive materials. Four common methods to attempt to decrease the dose usually have adverse effects on the image precision. First, decreasing the pixel dwell time will decrease the dose to the sample, but it will also decrease the signal collected at each pixel and decrease the SNR, possibly compromising the image precision. Second, decreasing the probe current will decrease the dose to the sample, but it will also have a similar effect as decreased dwell time. Third, increasing the pixel size by sampling the same area of the specimen with a coarser grid will decrease the dose to the sample, but it will also decrease the number of pixels per atomic column, possibly reducing the ability to locate the atomic column positions and compromising the image precision. Fourth, increasing the pixel size by decreasing the microscope's image magnification while keeping the same number of pixels in the image will decrease the dose to the sample, but it could have a similar effect as using fewer pixels. Here, we show that decreasing the electron dose by acquiring course-sampled HAADF STEM images can preserve high precision in locating atomic columns.

\section{Methods}

STEM experiments were performed on a probe aberrationcorrected FEI Titan microscope (FEI, Hillsboro, OR, USA) operated at $200 \mathrm{keV}$ with a 24.5 -mrad probe semi-angle, 24.5-pA probe current, and STEM resolution of approximately 0.9 Å. HAADF STEM images were collected using a detector range of 54 to $270 \mathrm{mrad}$ creating $Z$-contrast images where the atomic column intensity is approximately proportional to $Z^{1.7}$, where $Z$ is the atomic number of the atoms under the electron beam. The Au nanoparticle STEM sample was prepared by dispersing colloidal Au nanoparticles onto a non-porous 5-nm-thick Si membrane window grid. The colloidal Au nanoparticles had an average diameter of $5 \mathrm{~nm}$ and were synthesized using the phosphorus method in aqueous solution. Prior to STEM experiments, the sample was annealed under vacuum at $200^{\circ} \mathrm{C}$ for $48 \mathrm{~h}$ and then plasma-cleaned in a Fischione plasma cleaner in $25 \%$ oxygen-75\% argon mixture for approximately $5 \mathrm{~min}$ to reduce organic carbon surface contamination. A HAADF STEM image series consisting of 240 images was acquired with $256 \times 256$ pixels and a $5 \mu \mathrm{s} /$ pixel dwell time. A total dose of approximately 850 $\mathrm{C} / \mathrm{cm}$ [2] was used to acquire the whole image series. This dose is still high compared to what is possible with TEM [11], but for STEM, it is $1 \%$ of the dose previously used for sub-picometer precision on single-crystal samples and $19 \%$ of the dose used for a few picometer precision on a Pt nanoparticle [8]. The 240 image series was NR-registered $[8,9]$ and averaged to increase image SNR and to remove all sizes of distortions introduced by instabilities of the electron probe and sample during image series acquisition. The NR registration algorithm estimates sub-pixel shifts, but the registered images are resampled onto the original pixel grid using bilinear interpolation. The averaged image is then computed from the registered images.

To determine the position of the atomic columns in the image with sub-pixel accuracy, we fit a $4 \times 4$ pixel region around each atomic column to a twodimensional (2D) Gaussian function plus a constant defined by:

$$
\begin{aligned}
I(x, y)=I_{0}+A \exp & {\left[( \frac { - 1 } { 2 ( 1 - c ^ { 2 } ) } ) \left(\left(\frac{x-x_{0}}{x_{w}}\right)^{2}+\left(\frac{y-y_{0}}{y_{w}}\right)^{2}\right.\right.} \\
& \left.\left.-\left(\frac{2 c\left(x-x_{0}\right)\left(y-y_{0}\right)}{x_{w} y_{w}}\right)\right)\right]
\end{aligned}
$$

using the standard Levenberg-Marquart $L^{2}$-norm minimization method, similar to the methods of Van Aert et al. [12]. $x$ and $y$ are the positions for the intensity $I$, and the fitted parameters are $I_{0}, A, x_{w}, y_{w}, x_{0}, y_{0}$, and $c$. A Gaussian function was chosen for the fitting because the shape of the atomic column images is dominated by the incoherent source broadening, which is well approximated by a Gaussian function $[13,14]$.

The measure of precision $(\sigma)$ used here is the standard deviation in the measured interatomic separations, following Bals et al. [3]. Another measure of precision is the Gaussian fit error in locating atomic column positions if the image intensity is put in units of collected electrons and the Gaussian fit is weighted by the Poisson noise (square root of the number of collected electrons). Previously [8], we showed that in similar data sets, both these measures result in similar precisions, but the standard deviation is more sensitive to shifts in atomic column position imposed by STEM instabilities, making it a more appropriate measure for these results. Therefore, only the standard deviation measure will be discussed here. The images in all figures have not been rotated, so the 
horizontal and vertical directions are the $\operatorname{scan} x$ and $y$ directions, respectively. The image area at least five atomic planes from the nanoparticle surfaces in projection was used to calculate the precision to avoid the effect of surface atom bond length variation on the standard deviation of the measured interatomic separations. The image pixel size was calibrated so that the interatomic separations in this region match the known crystallographic separations. Because the nanoparticle analyzed here has a twin boundary defining two separate grains, the precision area was calculated separately in each grain at least five atomic planes from the boundary.

In order to measure the surface atom displacements, we constructed a perfect reference lattice that represents where the atomic columns would be if no surface bond length variation was present. The reference lattice was constructed by measuring the average lattice parameters in each grain separately from the image area used for precision analysis, then aligning the reference lattice to the image by minimizing the root mean squared displacement between the positions of the reference lattice and the atomic column positions of the precision area, and then extending the lattice to cover the whole nanoparticle. The vector difference between the measured position and the closest point on the reference lattice is the surface atom displacements.

\section{Results and discussion}

Figure 1a shows the first image of a 240 HAADF STEM image series of a $\mathrm{Au}$ nanoparticle along [10]. Figure $1 \mathrm{~b}$ shows the averaged image after the image series was NR-registered, displaying the enhanced SNR ratio compared to the first frame. Figure $1 \mathrm{~b}$ represents the average sample structure over the series acquisition time, and because of the long acquisition time, fast processes like thermal displacements and atom displacements from electron beam momentum transfer are not captured. Distortions from circular symmetry of the $\mathrm{Au}$ atomic columns are likely due to the electron probe's residual lower order aberrations. The $\mathrm{Au}$ nanoparticle displays two grains separated by a twin boundary. The atomic positions of the columns used for the precision analysis were determined by Gaussian fitting, as described in the previous section, and are shown by the red markers in Figure 2a,b for the top and bottom grains, respectively. The interatomic separations were calculated in the $a$ and $b$ directions for the top grain and the $c$ and $d$ directions for the bottom grain, defined by the directions marked in yellow in Figure 2a. The image precision $\sigma$, defined as the standard deviation of the interatomic separations $[8,3]$, is measured to be $\sigma_{a}=2.00 \mathrm{pm}, \sigma_{b}=1.96 \mathrm{pm}, \sigma_{c}=1.38 \mathrm{pm}$, and $\sigma_{d}=1.90 \mathrm{pm}$. Even though the pixel size in this data set is $59.93 \mathrm{pm} /$ pixel, and only a 4 pixel by 4 pixel area was used to do each atomic column fitting, 1 to $2 \mathrm{pm}$ precision is still achieved. Thus, NR registration of this data set allows atomic columns to be located with a precision of $3 \%$ of the pixel size. Picometer-scale precision in course sampled images enables the measurement of lattice strains to approximately $1 \%$ that are commonly associated with defects, interfaces, ion displacements, and surface relaxation at lower image magnification and lower dose than our previous work.

We measured the bond length variation of the $\mathrm{Au}$ nanoparticle as shown in Figure 3a,b,c. The red and blue markers in Figure 3a represent a perfect grid for the top and bottom grains where the atomic columns would be if no surface strains were present in the nanoparticle. Each atomic column of the nanoparticle was fit to a 2D Gaussian function as shown by the red markers in Figure $3 \mathrm{~b}$. The yellow arrows in Figure $3 \mathrm{~b}$ are magnified displacement vectors between the fit positions and the perfect grid, representing the bond length variation near the nanoparticle surface. Figure $3 \mathrm{c}$ shows a magnified image of the area within the green rectangle in Figure 3b showing more detail of the bond length variations. For reference, the displacement vector labeled by the letter
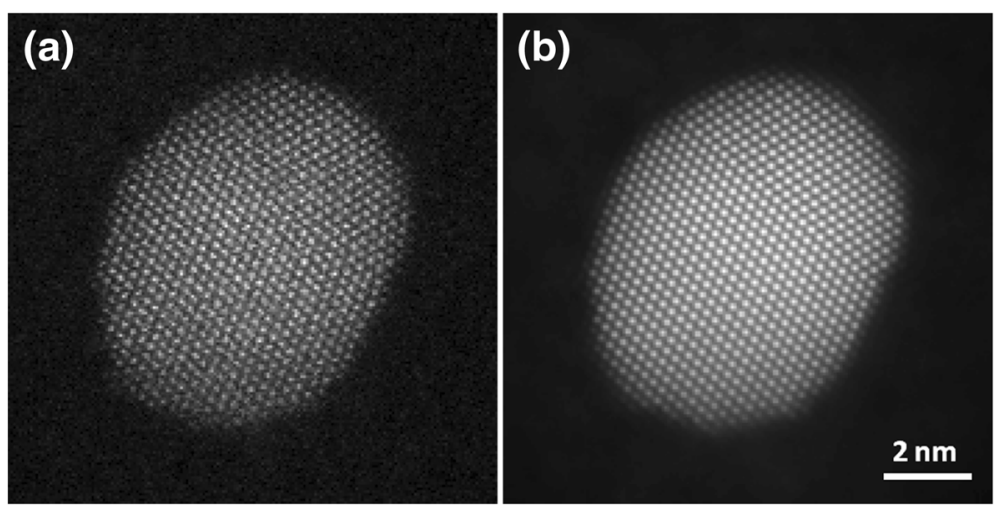

Figure 1 HAADF STEM image and averaged image after NR registration. (a) The first raw HAADF STEM image of the 240 image series of a Au nanoparticle along [10]. (b) The averaged image after the NR registration of the Au nanoparticle image series. 

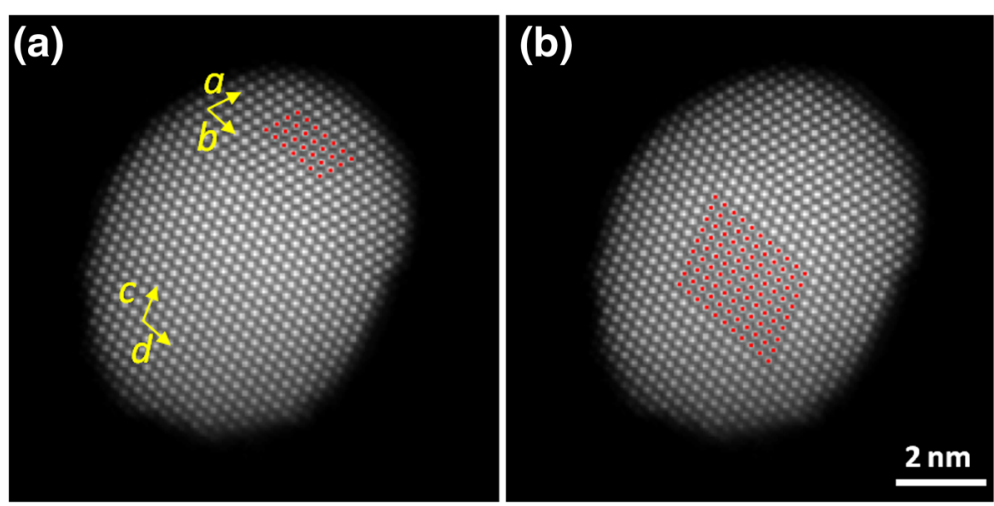

Figure 2 High-precision of the NR-registered and averaged image. The (a) top grain's and (b) bottom grain's precision area fit positions labeled by red markers. The yellow arrows signify the directions of the measured interatomic separations for the precision analysis.

A in Figure 3c represents $39 \mathrm{pm}$. The twin boundary is labeled by the blue arrow in Figure 3c. Because STEM produces projection images, we cannot extract the displacement component along the [10] beam direction. However, with this limitation, the Au nanoparticle displays only bond length contraction around its surface. Edge and corner atoms are observed to have larger bond length contraction than atoms present in the middle of surface facets. The observed bond length contraction is primarily towards the particle center with very little lateral displacement. As expected, there is no bond length variation along the twin boundary.

Our observations of bond length contraction of $\mathrm{Au}$ nanoparticles agree with theoretical and experimental results. The average bond length contraction at flat metal surfaces $[15,16]$ can be theoretically explained by the electronic surface charge rearranging to minimize the energy and creating an electrostatic force that shortens the bonds $[17,18]$ or by the lower coordination of surface atoms strengthening and shortening the remaining bonds [19]. Both explanations predict a larger bond length contraction of corner atoms near facet edges and steps, consistent with our observations. Our results agree with previous microscopic investigations of faceted Au NPs dispersed on graphene by Huang et al. using coherent electron nanodiffraction [20]. Their report of bond length contraction of surface atoms as large as $22 \mathrm{pm}$ for edge and corner bonds is in reasonable agreement with our observations.

Because high-precision STEM and TEM imaging usually involves taking many images of the same structure to increase SNR, it involves a large electron dose to the sample. For beam-sensitive materials, high-precision imaging is therefore a problem and sometimes not possible. Any means to decrease electron dose and still get the quantitative information from a sample is helpful. Here, we show that increasing the STEM image pixel size almost as large as possible while distinguishing atomic columns still allows for picometer-scale image precision using NR registration. The large pixels from lowering the magnification decreases the electron dose to the sample, reducing electron beam damage. Coarse sampling also allows for imaging larger sample areas and potentially larger structures of interest. This could be used for imaging whole nanoparticles with picometer-scale precision (as shown here) or imaging other larger structure such as
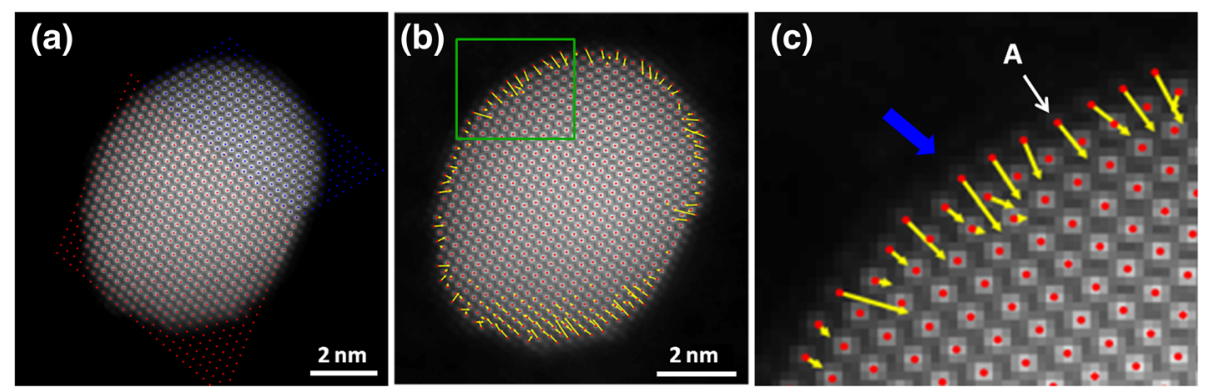

Figure 3 The bond length variation of the surface atoms in the Au nanoparticle. (a) The NR-registered and averaged image with the top and bottom grain's perfect grid labeled by blue and red markers, respectively. (b, $\mathbf{c})$ The NR-registered and averaged image with the fit positions labeled by red markers and the magnified displacement vectors marked by the yellow arrows. (c) A magnified image of the area within the green box in (b) with the twin boundary marked by the blue arrow. 
surfaces, extended defects, far-reaching strain fields, and interfaces.

Another strategy to reduce dose is to reduce the number of images that are registered and averaged. In our previous work, precision saturated approximately half way through the data series [8]. That analysis has not been duplicated here; but in general, experiments using NR registration of identical frames can trade off dose for SNR and precision after acquisition depending on the magnitude of the displacements of interest and the beam sensitivity of the sample.

\section{Conclusions}

In conclusion, 1- to 2-pm image precision has been achieved by non-rigidly registering and averaging a series of short-exposure course-sampled HAADF STEM images of a 5- to 6-nm Au nanoparticle. These imaging conditions substantially reduce the dose to the sample by having a large pixel size and low exposure time, while still achieving high precision in locating atomic columns. Non-rigid registration also allows for reduced magnification, capturing the whole $\mathrm{Au}$ nanoparticle in one high-precision image. The high-precision image reveals only bond length contraction around the nanoparticle surface and no bond length variation along a twin boundary that separates the nanoparticle into two grains. Atoms present at the surface edges and corners show larger bond length contraction than atoms near the center of surface facets, consistent with theory and previous microscopic investigations.

\section{Abbreviations}

2D: two-dimensional; HAADF: high-angle annular dark field; NR: non-rigid: SNR: signal-to-noise ratio; STEM: scanning transmission electron microscopy; TEM: transmission electron microscopy.

\section{Competing interests}

The authors declare that they have no competing interests.

\section{Authors' contributions}

PMV conceived the study of STEM precision and nanoparticle surface relaxation. $A B Y$ performed the experiments and data analysis. $B B, W D$, and $P B$ developed and applied the NR registration algorithm. ABY drafted the manuscript, and all authors discussed and revised it. All authors read and approved the final manuscript.

\section{Acknowledgements}

The authors at UW-Madison acknowledge the funding from the Department of Energy, Basic Energy Sciences (DE-FG02-08ER46547) for microscopy experiments and analysis. UW-Madison facilities and instrumentation are supported by the UW Materials Research Science and Engineering Center (DMR-

1121288). The authors at USC acknowledge the funding from the NSF grant DMS-1222390, the Interdisciplinary Mathematics Institute, and the College of Arts and Sciences. The authors at RWTH Aachen were funded in part by the Excellence Initiative of the German Federal and State Governments.

\section{Author details}

${ }^{1}$ Department of Materials Science and Engineering, University of Wisconsin-Madison, 1509 University Ave, Madison, WI 53706, USA. ${ }^{2}$ Aachen Institute for Advanced Study in Computational Engineering Science (AICES), RWTH Aachen, Schinkelstr. 2, 52062 Aachen, Germany. ${ }^{3}$ Interdisciplinary Mathematics Institute, University of South Carolina, 1523 Greene Street,
Columbia, SC 29208, USA. ${ }^{4}$ Institut für Geometrie und Praktische Mathematik, RWTH Aachen, Templergraben 55, 52056 Aachen, Germany.

Received: 21 October 2014 Accepted: 27 January 2015

Published online: 25 March 2015

\section{References}

1. Haider, M, Uhlemann, S, Schwan, E: Electron microscopy image enhanced. Nature. 392, 768-769 (1998)

2. Jia, CL, Thust, A: Investigation of atomic displacements at a S3 $\{111\}$ twin boundary in $\mathrm{BaTiO} 3$ by means of phase-retrieval electron microscopy. Phys. Rev. Lett. 82, 5052-5055 (1999)

3. Bals, S, Van Aert, S, Van Tendeloo, G, Ávila-Brande, D: Statistical estimation of atomic positions from exit wave reconstruction with a precision in the picometer range. Phys. Rev. Lett. 96, 096106 (2006)

4. Jia, C-L, Mi, SB, Urban, K, Vrejoiu, I, Alexe, M, Hesse, D: Atomic-scale study of electric dipoles near charged and uncharged domain walls in ferroelectric films. Nat. Mater. 7, 57-61 (2008)

5. Kimoto, K, Asaka, T, Yu, X, Nagai, T, Matsui, Y, Ishizuka, K: Local crystal structure analysis with several picometer precision using scanning transmission electron microscopy. Ultramicroscopy. 110, 778-782 (2010)

6. Kim, Y-M, He, J, Biegalski, MD, Ambaye, H, Lauter, V, Christen, HM, Pantelides, ST, Pennycook, SJ, Kalinin, SV, Borisevich, AY: Probing oxygen vacancy concentration and homogeneity in solid-oxide fuel-cell cathode materials on the subunit-cell level. Nat. Mater. 11, 888-894 (2012)

7. Van Aert, S, den Dekker, AJ, Van Dyck, D, van den Bos, A: Optimal experimental design of STEM measurement of atom column positions. Ultramicroscopy 90, 273-289 (2002)

8. Yankovich, AB, Berkels, B, Dahmen, W, Binev, P, Sanchez, SI, Bradley, SA, Li, Ao, Szlufarska, I, Voyles, PM: Picometre-precision analysis of scanning transmission electron microscopy images of platinum nanocatalysts. Nat. Commun. 5,4155 (2014)

9. Berkels, B, Binev, P, Blom, DA, Dahmen, W, Sharpley, RC, Vogt, T: Optimized imaging using non-rigid registration. Ultramicroscopy. 138, 46-56 (2013)

10. LeBeau, JM, Findlay, SD, Allen, L, Stemmer, S: Standardless atom counting in scanning transmission electron microscopy. Nano Lett. 10, 4405-4408 (2010)

11. Kisielowski, C, Wang, L-W, Specht, P, Calderon, HA, Barton, B, Jiang, B, Kang, JH, Cieslinski, $R$ : Real-time sub-Ångstrom imaging of reversible and irreversible conformations in rhodium catalysts and graphene. Phys. Rev. B. 88,024305 (2013)

12. Van Aert, S, Verbeeck, J, Erni, R, Bals, S, Luysberg, M, Van Dyck, D, Van Tendeloo, G: Quantitative atomic resolution mapping using high-angle annular dark field scanning transmission electron microscopy. Ultramicroscopy 109, 1236-1244 (2009)

13. Dwyer, C, Erni, R, Etheridge, J: Measurement of effective source distribution and its importance for quantitative interpretation of STEM images. Ultramicroscopy. 110, 952-957 (2010)

14. LeBeau, J, Findlay, S, Allen, L, Stemmer, S: Quantitative atomic resolution scanning transmission electron microscopy. Phys. Rev. Lett. 100, 1-4 (2008)

15. Bohnen, KP, Ho, KM: Structure and dynamics at metal surfaces. Surf. Sci. Rep. 19, 99-120 (1993)

16. Zambelli, T, Wintterlin, J, Trost, J, Ertl, G: Identification of the "active sites" of a surface-catalyzed reaction. Science (80) 273, 1688-1690 (1996)

17. Finnis, MW, Heine, V: Theory of lattice contraction at aluminium surfaces. J. Phys. F Met. Phys. 4, L37-L41 (1974)

18. Smoluchowski, R: Anisotropy of the electronic work function of metals. Phys. Rev. 60, 661-674 (1941)

19. Pauling, L: Atomic radii and interatomic distances in metals. J. Am. Chem. Soc. 69, 542-553 (1947)

20. Huang, WJ, Sun, R, Tao, J, Menard, LD, Nuzzo, RG, Zuo, JM: Coordination-dependent surface atomic contraction in nanocrystals revealed by coherent diffraction. Nat. Mater. 7, 308-313 (2008) 